Aims. Thriving on the pressure of "publish or perish" experienced by academicians, the industry of predatory publishers with dubious quality has mushroomed and gained their notoriety. The battle of uncovering predatory publishers, including Beall's list, has proven to be tough given the huge monetary gain generated by the predatory publishers. It may be difficult for an inexperienced junior researcher to identify those predatory publishers' soliciting emails, which may disguise as a reputable journal's articlecommissioning process. To date, there is a limited systematic approach to identify such emails. Hence, this research is aimed to describe the common features of soliciting emails from publishers which appeared to be predatory.

Method. This self-study involved reviewing the content of emails in the spam folder of authors, a team of junior researchers in psychiatry, for a month. Emails included in this study were soliciting emails relevant to publications and the following were reviewed: types of solicitation, sentences used, strategies used, and information available in the public domain of their webpages. Informative types of emails were excluded.

Result. The solicitation could include: 1 ) request for a manuscript to be published a journal article, 2) request for a thesis to be published as a book, 3) request to write for a book chapter, 4) invitation to be an editorial member or a reviewer with the offer of free publishing, 5) invitation to be a speaker for a conference, and 6) proofreading services. The publisher may cite a published article of the author from another journal, which was the source where they identified the author's email. Common strategies used for solicitation included: 1) promising a fast-tracked and guaranteed publication, 2) using compliments that appeared to be inappropriate, 3) repetitive emails, and 4) using argumentum ad passiones to induce guilt. The common features of the webpages of those publishers included: 1) open access publishing as the only option, 2) extensive list of indexing services excluding wellestablished indexing agencies, and 3 ) the publisher has a huge collection of journals in different disciplines.

Conclusion. It is hoped that these findings will help junior researchers in psychiatry to stay vigilant to avoid falling into the trap of predatory publishers, which may result in financial loss and loss of work to plagiarism. Total eradication of those predatory soliciting emails is unlikely despite the advancement of spam filtering technology, which necessitates a more united effort from different stakeholders to come out with a probable solution.

\section{Virtual reality exposure therapy in panic disorder: a pilot study}

Clara Gitahy Falcão Faria ${ }^{1 \star}$, Veruska Andrea Santos ${ }^{1}$, Marcos Fidry Muniz ${ }^{1}$, Mariana Costa do Cabo ${ }^{1}$, Antonio Egidio Nardi ${ }^{2}$ and Rafael Chistophe da Rocha Freire ${ }^{3}$

${ }^{1}$ Institute of Psychiatry, Federal University of Rio de Janeiro;

${ }^{2}$ Institute of Psychiatry, Federal University of Rio de Janeiro, Full Professor of Psychiatry, Federal University of Rio de Janeiro and

${ }^{3}$ Associate professor, Queen`s University

${ }^{*}$ Corresponding author.

doi: 10.1192/bjo.2021.672

Aims. To ascertain if virtual reality exposure therapy (VRET) is an effective add-on tool in the treatment of Panic Disorder (PD). Background. The exposure to virtual stimuli has been studied as a useful treatment for PD. However, the studies with PD are still scarce and use dissimilar protocols, with effectiveness varying according to the protocol applied.

Method. Eight PD patients received VRET as an add-on treatment to pharmacotherapy. The treatment protocol consisted of eight sessions. The first session is for the patient to understand the treatment and to answer the questionnaires. The second and third sessions were to prepare the patients for exposures with breathing training using diaphragmatic breathing and others breathing techniques to manage anxiety. From the fourth to eighth sessions, the patients followed a hierarchy of tasks during virtual reality exposure. Clinicians rated the Clinical Global Impression Scale (CGI) and the Panic Disorder Severity Scale (PDSS). The patients rated the Diagnostic Symptom Questionnaire (DSQ); the Mobility Inventory (MI), the Anxiety Sensibility Index (ASI-R), the Beck Depression Inventory (BDI), the Beck Anxiety Inventory (BAI) and the WHOQOL-BREF before and after the protocol. After all exposures, the Igroup Presence Questionnaire (IPQ) was applied to measure the sense of presence experienced in the virtual environment. The virtual environment simulated the subway of Rio de Janeiro.

Result. There were no statistically significant improvements in the CGI-S, PDSS, BAI, MI or WHOQOL. There was a significant improvement in the BDI scores $(\mathrm{P}=0.033)$. There was a trend towards improvement of anxiety measured by the ASI-R $(\mathrm{P}=0.084)$ and of panic symptoms measured by the DSQ $(\mathrm{P}=0.081)$ scores. There was also a significant improvement of sense of presence (IPQ - general presence) through the exposure sessions.

Conclusion. Our study demonstrated that VRET as an add-on to pharmacological therapy could benefit PD patients. Despite the lack of significant differences in the means, the dispersion of PDSS and BAI scores were smaller after treatment compared to before treatment, suggesting that patients with more severe anxiety, panic and agoraphobia symptoms benefited more of the VRET protocol so, at the end of the treatment, differences were found in important measures of panic. Randomized controlled clinical trials are warranted to confirm the efficacy of VRET.

This study was funded by the Brazilian National Council for Scientific Development (Cnpq). The authors report no conflicts of interest.

\section{ECT practice in England from 2012/13 to 2018/19: a retrospective analysis}

Mohan Gondhalekar ${ }^{1 \star}$, Robert Chaplin ${ }^{2}$, Sinead Rogers ${ }^{3}$ and Oriana Delgado ${ }^{4}$

${ }^{1}$ Royal College of Psychiatrists, Consultant Old Age Psychiatrist and Clinical Fellow, Older Adult Mental Health Team Auckland District Health Board; ${ }^{2}$ Royal College of Psychiatrists, Research Fellow and Consultant General Adult Psychiatrist; ${ }^{3}$ Deputy Programme Manager ECTAS CCQI RCPsych and ${ }^{4}$ Royal College of Psychiatrists

${ }^{\star}$ Corresponding author.

doi: $10.1192 /$ bjo.2021.673

Aims. The purpose of this study was to look longitudinally at ECT practice in England over the past 7 years: namely over the following key time periods; 2012/13, 2014/15, 2016/17, 2017/18, and 2018/19. A previous study by Chaplin et al, published in 2016, found that there had been a striking decline observed in the number of courses of ECT prescribed to patients from 2006 to two time points i.e. $2012 / 13$ and $2014 / 15$.

In this study we investigated whether or not this trend had continued. Hence we looked at the change in frequency of ECT use, the length of ECT courses, patient demographics and clinical outcomes; between 2012/13 and 2018/19.

Background. Electroconvulsive therapy (ECT) is an effective treatment for Major Depression, Treatment-Resistant Depression, Catatonia, and Clozapine-resistant psychosis. There 
have been regular improvements in the administration of ECT, over the past two decades. Increases in the volume of the hippocampus and the amygdala have consistently been observed in ECT studies. Stigma has been the major barrier to patients receiving ECT in a timely fashion. The Royal College of Psychiatrists (RCPsych) Centre for Quality Improvement (CCQI) established the ECT Accreditation Service (ECTAS) back in 2006. ECTAS had the aim of standardising ECT practice through the production of evidence-based standards that all member ECT Clinics could use to support their practice.

Method. We looked at the minimum dataset of information collected from ECTAS Members within England for the following years; 2012/13, 2014/15, 2016/17, 2017/18, 2018/19. In 2012/13, 2325 adjusted courses of ECT treatment were given to patients in England. In 2014/15 it was 2302.

Result. Between 2012/13 and 2018/19; two thirds of ECT patients continue to be female. The modal age of patients has also remained the same at 70 years. The number of patients detained under the Mental Health Act 1983 receiving ECT has gone up by $12 \%$; suggesting that the patients receiving ECT were more clinically unwell. After treatment, CGI scale scores (i.e. the very much improved and much improved scores) slightly reduced by $6 \%$ from $2012 / 3$ to $2018 / 19$.

Conclusion. The use of ECT in England notably declined from 2006 to $2012 / 13$ and 2014/15. However, from 2012/13 to 2018/ 19 , ECT use has remained relatively stable; suggesting that it is currently being used appropriately on patients, who are amongst the most severely unwell. The clinical effectiveness of ECT remains high however, it has slightly dipped by $6 \%$.

\section{"Prevalence of orthorexia nervosa in a sample of patients attending Sligo/Leitrim mental health services with a diagnosis of eating disorder"}

Ignazio Graffeo*, Mary Harron and Edmond O’Mahony

Sligo/Leitrim Mental Health Services - HSE

${ }^{*}$ Corresponding author.

doi: 10.1192/bjo.2021.674

Aims. The main aim of this study is to investigate its presence in a sample of patients already diagnosed with a canonical eating disorder and also to understand eventual overlaps with other clinical disorders in order to optimize treatment and follow-up. The ORTO-15 questionnaire, developed by an Italian team of researchers in 2005, was used to achieve the above aims: it is a tool comprehensive of 15 questions that assesses eating habits perceived as healthy. Really interesting and fascinating is to comprehend if people with a diagnosis of eating disorder present orthorectic behaviour and how this emerging reality fits in the Irish society with its peculiarities and uniqueness.

Method. Every patient was asked to complete a demographic grid (elaborated by the researchers, which includes information regarding: age, gender, race, weight, height, hours of weekly exercise, years of education, employment situation, medical illnesses, smoking habits, type of diet, average weekly alcohol intake) and the Orto-15 questionnaire

Result. The Point Prevalence obtained is $17.9 \%$.

Conclusion. The results obtained from this study give a clear indication of the profile of the orthorexic patient, considered that the sample was obtained from a population of people with a diagnosis of Eating Disorder:

Caucasian woman in her 30 s

Exercising 5 hours per week

Secondary education
Unemployed

Non-smoker

Diagnosis of Anorexia Nervosa

No other comorbid psychiatric illnesses

Standard pattern of eating

Minimal or absent alcohol consumption

Normal range BMI

According to previous Italian studies (Ramacciotti et al. 2011), the expected rates of Orthorexia Nervosa in the general population are between $6.9 \%$ and $57.6 \%$, with a peak of $81.8 \%$ in specific populations, fact that places our examined sample in the lower side of the prevalence previously considered. It is very difficult to comprehend and explain the reasons behind this fact and probably this is due to an overshadowing of symptoms with the major eating disorders. It is also significant the absence of correlation found between OCD and $\mathrm{ON}$ and also the fact that $\mathrm{ON}$ is more linked to Bulimia Nervosa in our sample rather then Anorexia Nervosa.

What happens to frequent attenders when they attend psychiatric liaison services? a clinical and demographic profile

Frederick Grose ${ }^{\star}$, Tennyson Lee, Richelle Canlas, William Phung, Rikke Albert, Alana Ahmet and Jia Song

East London NHS Foundation Trust

${ }^{\star}$ Corresponding author.

doi: 10.1192/bjo.2021.675

Aims. We aimed to describe the demographic and clinical profile, and management of frequent attenders to a psychiatric liaison service.

Background. Frequent Attenders to emergency departments contribute significantly to the burden on health services and by definition are subjectively highly stressed. It is therefore important that mental health services develop effective responses to this group of patients. A systematic literature search indicated a paucity of information on this group of patients.

Method. We conducted a case series of 49 frequently attending patients to the Psychiatric Liaison service in Tower Hamlets, East London NHS Foundation Trust.

We defined frequent attenders as seeing the Psychiatric Liaison Service 5 or more times in 2018. We excluded 4 patients aged $<18$ years or $>65$ years.

For each patient we collected data regarding their demographics; the details of each attendance to the Psychiatric Liaison Service; and their use of other psychiatric services.

We then conducted a multivariate analysis, including stratification of patients based on number of attendances to identify correlation between frequency of attendance and the other information.

Result. Demographic: The 45 patients reviewed had a mean age of 37 and a mean of 7 attendances during the study period.

Clinical: $89 \%$ had a history of emotional trauma, $71 \%$ of substance misuse, and $49 \%$ of any personality disorder. Only $9 \%$ of the patients were under the care of the locality Personality Disorder Service.

$73 \%$ of the patients were under the care of any other psychiatric service. There was no correlation between being under other services and the frequency of attendance.

Only $31 \%$ had contact with the locality Frequent Attenders Service during the study period, as this was established recently. 